Stockholm

November 1994

\title{
NOTE ON MASSIVE SPIN 2 IN CURVED SPACE
}

\author{
Ingemar Bengtsson凹 \\ Fysikum \\ University of Stockholm \\ Box 6730, S-113 85 Stockholm, Sweden
}

\begin{abstract}
We give a Hamiltonian formulation of massive spin 2 in arbitrary Einstein space-times. We pay particular attention to Higuchi's forbidden mass range in deSitter space.
\end{abstract}

\footnotetext{
${ }^{1}$ Email address: ingemar@vana.physto.se
} 


\section{INTRODUCTION AND CONCLUSIONS.}

It may be that Nature evades the consistency problems of the higher spin equations through the simple expedient of not employing them at all. Even so, and certainly as long as we are not quite sure of Nature's verdict, the subject remains notoriously fascinating. Here we will study the massive spin 2 model generalized to space-times whose metric obeys Einstein's vacuum equations, but which are otherwise arbitrary. It is well known that the Fierz-Pauli equations are algebraically consistent whenever the background metric has the Einstein property. What is less well known is a peculiar phenomenon in deSitter space, first studied by Higuchi [1] (following an observation originally made by Deser and Nepomechie [2]).

Let us recall what the situation is. In flat space, the Fierz-Pauli equations are known to describe a field with five degrees of freedom, which carries an irreducible massive spin 2 representation of the Poincaré group. These equations remain algebraically consistent in curved space-times whose metric solves Einstein's vacuum equations. (This does not mean that a single massive spin 2 field can be coupled to gravity, in fact the consensus is that it can not [4]. Massive spin 2 fields do appear among the interacting modes of Kaluza-Klein and string theories, however.) When a positive cosmological constant is included, it was observed [2] that the equations possess a gauge invariance when $3 m^{2}=2 \lambda$. This was further elucidated by Higuchi [1], who found that the quantum version of the theory, in deSitter space, has negative norm states whenever $m^{2}$ lies below that value. This underlies the further interesting fact [1] that there is no van Dam-Veltman discontinuity [5] in deSitter space. The result can be understood from the point of view of the representation theory of the deSitter group [6]. Here we will understand this phenomenon from yet another, purely classical, point of view, by verifying explicitly that the Hamiltonian is not positive definite when $m^{2}$ belongs to the forbidden mass range.

To be precise, the equations that we will study are

$$
W_{\alpha \beta}-\frac{1}{2} g_{\alpha \beta} W_{\gamma}^{\gamma}+\frac{4 \lambda}{D-2} h_{\alpha \beta}-\frac{2 \lambda}{D-2} g_{\alpha \beta} h=m^{2}\left(h_{\alpha \beta}-g_{\alpha \beta} h\right),
$$

where $D$ is the dimension of spacetime, $h \equiv h_{\alpha}^{\alpha}$,

$$
W_{\alpha \beta} \equiv \nabla_{\gamma} \nabla^{\gamma} h_{\alpha \beta}-\nabla_{\gamma} \nabla_{\alpha} h_{\beta}^{\gamma}-\nabla_{\gamma} \nabla_{\beta} h_{\alpha}^{\gamma}+\nabla_{\alpha} \nabla_{\beta} h,
$$

and $\nabla_{\alpha}$ is the covariant derivative compatible with the the background metric $g_{\alpha \beta}$, which obeys Einstein's vacuum equations

$$
R_{\alpha \beta}=\frac{2 \lambda}{D-2} g_{\alpha \beta} .
$$

Eq. (11) implies that $h_{\alpha \beta}$ is traceless and obeys the Fierz-Pauli equations

$$
\nabla_{\gamma} \nabla^{\gamma} h_{\alpha \beta}+\frac{4 \lambda}{D-2} h_{\alpha \beta}=m^{2} h_{\alpha \beta} \quad \quad \nabla^{\beta} h_{\alpha \beta}=0 .
$$


This is algebraic consistency. It is natural to demand also that the Hamiltonian should be bounded from below, in suitably chosen backgrounds. This is the main question to be discussed here.

The above derivation of the Fierz-Pauli equations breaks down for the special value $(D-1) m^{2}=2 \lambda$. What happens then [2] is that eq. (1) is invariant under the gauge transformation

$$
\delta h_{\alpha \beta}=\nabla_{\alpha} \nabla_{\beta} \xi+\frac{2 \lambda}{(D-1)(D-2)} g_{\alpha \beta} \xi .
$$

In section 2 we set up the Hamiltonian version of the above equations in arbitrary Einstein spaces. For flat space, this was first done in ref. [3]; we have streamlined the derivation through the simple expedient of relying on results for linearized gravity as far as possible. In section 3 we study the positivity properties of the Hamiltonian in flat and deSitter backgrounds. It is shown (not surprisingly, perhaps) that Higuchi's forbidden mass range can be characterized as that range of mass for which the Hamiltonian is not bounded from below, thus emphasizing that the phenomenon has nothing to do with quantum mechanics as such. In section 4 we briefly consider, and rule out, some deformations of the gauge algebra (5) that appears for $3 m^{2}=2 \lambda$. The conclusion of this analysis is that the simplest attempts to write down a non-linear generalization of this algebra fail. It is therefore unlikely that there exists an interesting non-linear version of this somewhat peculiar gauge theory.

The mass of the lightest (unstable) spin 2 meson is $1275 \mathrm{MeV}$ - the square root of the cosmological constant, in the same units, is known to be very much smaller indeed, which certainly suggests that Higuchi's forbidden mass range is of no real interest. Nevertheless we feel that it is worth some attention as part of the lore of higher spins. As a speculation, we observe that the exceptional value $3 m^{2}=2 \lambda$ leads to a model which not only has an extra gauge invariance, but which also exhibits propagation confined to the light cone [2]. Could this serve as a building block for an unknown long-range force?

\section{HAMILTONIAN FORMULATION.}

It is not an attractive task to perform a $3+1$ decomposition of the action from which eq. (1) can be derived. A short cut is to set $m^{2}=0$ at first, in which case all we have to do is to linearize the ADM action for gravity. The ADM action is

$$
S_{A D M}=\int \dot{\mathrm{g}}_{a b} \Pi^{a b}-\mathbf{N} \mathcal{H}-\mathbf{N}^{a} \mathcal{H}_{a}
$$

where

$$
\begin{gathered}
\mathcal{H}=\frac{1}{\sqrt{\mathbf{g}}}\left(\mathbf{g}_{a c} \mathbf{g}_{b d}-\frac{1}{D-2} \mathbf{g}_{a b} \mathbf{g}_{c d}\right) \Pi^{a b} \Pi^{c d}-\sqrt{\mathbf{g}}(R(\mathbf{g})-2 \lambda) \\
\mathcal{H}_{a}=-2 \nabla_{b} \Pi^{b}{ }_{a} .
\end{gathered}
$$


We linearize this around a solution of the equations, according to

$$
\begin{array}{rlrl}
\mathbf{g}_{a b} & \equiv g_{a b}+h_{a b} & \Pi^{a b} \equiv \pi^{a b}+p^{a b} \\
\mathbf{N} \equiv N+n & \mathbf{N}^{a} \equiv N^{a}+n^{a}
\end{array}
$$

Note that, from now on, we will use the spatial background metric $g_{a b}$ to raise and lower spatial vector indices, $h \equiv h_{a}^{a}$, and $\pi^{a b}$ is also part of the fixed background. Also, the Ricci tensor and the covariant derivative that will occur below are defined with respect to the background metric.

Proceeding in this way, we obtain the following action for linearized gravity:

$$
S=\int \dot{h}_{a b} p^{a b}-N \mathcal{H}^{(2)}-N^{a} \mathcal{H}^{(2)}-n \mathcal{H}^{(1)}-n^{a} \mathcal{H}^{(1)}
$$

The constraints of the linearized theory are explicitly

$$
\begin{gathered}
\mathcal{H}^{(1)}=\frac{2}{\sqrt{g}}\left(\pi_{a b} p^{a b}-\frac{1}{D-2} \pi p+\pi^{a c} \pi_{c}{ }^{b} h_{a b}-\frac{1}{D-2} \pi \pi^{a b} h_{a b}\right)- \\
-\sqrt{g}\left(\nabla_{a} \nabla_{b} h_{a}{ }^{b}-\nabla_{a} \nabla^{a} h-R^{a b} h_{a b}+R h-2 \lambda h\right) \approx 0 . \\
\mathcal{H}_{a}^{(1)}=-2 \nabla_{b} p^{b}{ }_{a}+\pi^{b c}\left(\nabla_{a} h_{b c}-2 \nabla_{b} h_{c a}\right) \approx 0 .
\end{gathered}
$$

The Hamiltonian of the linearized theory is quadratic in the fields $h_{a b}$ and $p^{a b}$. It can be obtained from the ADM phase space action through a straightforward but lengthy calculation. Unfortunately the explicit formula that one obtains is also lengthy, and not illuminating to look at, so we do not give it here. In fact, the entire, explicit formula will not be needed in the sequel. So we simply write the Hamiltonian of linearized gravity as

$$
H \equiv \mathcal{H}^{(2)}[N]+\mathcal{H}_{a}^{(2)}\left[N^{a}\right] \equiv \int N \mathcal{H}^{(2)}+N^{a} \mathcal{H}_{a}^{(2)}
$$

Note that $N$ and $N^{a}$ are part of the fixed background; this equation also serves to introduce our notation for smearing with test function.

For all backgrounds that solve Einstein's equations, the linearized constraint algebra which is abelian - can be found through linearization of the constraint algebra of the nonlinear theory. Moreover, by studying the second order terms of the non-linear constraint algebra one can deduce that

$$
\begin{aligned}
& \frac{d}{d t} \mathcal{H}^{(1)}[n]=\frac{\partial}{d t} \mathcal{H}^{(1)}[n]+\left\{\mathcal{H}^{(1)}[n], H\right\}= \\
& \quad=\mathcal{H}_{a}^{(1)}\left[n \nabla^{a} N-N \nabla^{a} n\right]-\mathcal{H}^{(1)}\left[N^{a} \nabla_{a} n\right] \approx 0 .
\end{aligned}
$$


Hence the linearized version of the Hamiltonian constraint is conserved under time evolution, as is indeed required by the consistency of linearized gravity. Eq. (15) - which would have been awkward to prove through directly, using an explicit form of the Hamiltonian - will be used below.

With linearized gravity in hand, the Hamiltonian formulation of the massive theory can be derived in a few steps. First we add a Pauli-Fierz mass term to the action:

$$
S_{m} \equiv \int \sqrt{-g}\left(h_{\alpha \beta} h^{\alpha \beta}-h h\right)
$$

Now, by comparing to the ADM decomposition of the space-time metric, it is easy to see that eqs. (9 - 10) implies the following "names" for the time-time and time-space components of the field $h_{\alpha \beta}$ that occur here:

$$
\begin{gathered}
h_{t a}=n_{a}+N^{b} h_{b a} \\
h_{t t}=-2 n N+2 n_{a} N^{a}+N^{a} h_{a b} N^{b} .
\end{gathered}
$$

It is then a matter of straightforward calculation to show that

$$
\sqrt{-g}\left(h_{\alpha \beta} h^{\alpha \beta}-h h\right)=N \sqrt{g}\left(h_{a b} h^{a b}-h^{2}\right)-\frac{2 \sqrt{g}}{N} n_{a} n^{a}-4 \sqrt{g} n h .
$$

In this form, these terms are to be added to the linearized gravity phase space action (11). We observe that the Lagrange multiplier $n^{a}$ then occurs quadratically, so that it can be solved for. Inserting the result back into the action, we are then left with

$$
\begin{aligned}
S=\int & \dot{h}_{a b} p^{a b}-N \mathcal{H}^{(2)}-\frac{m^{2} N}{4} \sqrt{g}\left(h_{a b} h^{a b}-h^{2}\right)- \\
& -\frac{N}{2 m^{2} \sqrt{g}} \mathcal{H}_{a}^{(1)} \mathcal{H}^{(1) a}-n\left(\mathcal{H}^{(1)}-m^{2} \sqrt{g} h\right) .
\end{aligned}
$$

If any other form of the mass term than that given by Fierz and Pauli is being used one finds that also $n$ occurs quadratically here. When this Lagrange multiplier is eliminated from the action one finds a model with no constraints on the six degrees of freedom. The "extra" degree of freedom is a negative energy ghost in Minkowski space (where such a statement makes sense).

We must now compute the secondary constraints, if any, by taking the total time derivative of the constraint that is obtained when the action is varied with respect to the Lagrange multiplier $n$. It is here that eq. (15) comes in handy. After a non-trivial but not too lengthy calculation, one finds in this way that the degrees of freedom are subject to the following constraints:

$$
\begin{gathered}
\Psi_{1}=\mathcal{H}^{(1)}-m^{2} \sqrt{g} h \approx 0 \\
\Psi_{2}=-\frac{1}{2} \nabla^{a} \mathcal{H}_{a}^{(1)}+\frac{m^{2}}{D-2} p-m^{2} \frac{D-4}{D-2} \pi^{a b} h_{a b} \approx 0 .
\end{gathered}
$$


The algebra of these constraints is

$$
\left\{\Psi_{1}(x), \Psi_{2}\left(x^{\prime}\right)\right\}=-m^{2}\left(m^{2}-\frac{2 \lambda}{D-1}\right) \frac{D-1}{D-2} \sqrt{g} \delta\left(x, x^{\prime}\right) .
$$

We see that the constraints are second class whenever

$$
(D-1) m^{2} \neq 2 \lambda \text {. }
$$

For all such values of $m^{2}$ then, the model possesses the expected five degrees of freedom. The special value of $\mathrm{m}^{2}$ for which the constraint algebra is first class (and the number of degrees of freedom drops to four) is precisely the value found in ref. [2] - as had to be the case, of course.

\section{POSITIVITY PROPERTIES.}

For suitable background space-times, we expect the Hamiltonian to be bounded from below. To show this one will have to employ a decomposition of the field variable into irreducible parts, which in general is not straightforward. Therefore we confine our attention to deSitter space, and use the coordinate system

$$
d s^{2}=-d t^{2}+e^{2 \alpha t}\left(d x^{2}+d y^{2}+d z^{2}\right)
$$

where

$$
(D-1) \alpha^{2}=\frac{2 \lambda}{D-2} .
$$

Then we can use flat space projection operators to perform the decomposition, viz.

$$
\begin{array}{ll}
h_{a b}^{(2)}=\left(\Pi_{a}^{(c} \Pi_{b}^{d)}-\frac{1}{3} \Pi_{a b} \Pi^{c d}\right) h_{c d} & h_{a b}^{(0)}=\frac{1}{3} \Pi_{a b} \Pi^{c d} h_{c d} \\
h_{a b}^{(1)}=\left(\Pi_{a}^{(c} \Omega_{b}^{d)}+\Omega_{a}^{(c} \Pi_{b}^{d)}\right) h_{c d} & h_{a b}^{\left(0^{\prime}\right)}=\Omega_{a b} \Omega^{c d} h_{c d},
\end{array}
$$

where

$$
\Pi_{a}^{b}=\delta_{a}^{b}-\frac{1}{\triangle} \partial^{a} \partial^{b} \quad \Omega_{a}^{b}=\frac{1}{\triangle} \partial_{a} \partial^{b}
$$

$\triangle$ is the Laplacian, and the spatial dimension was set to three for simplicity $(D=$ 4). Actually there is a problem here, because in deSitter space the fall-off behaviour of the fields is not that which is required to justify our manipulations with the projection operators. However, we can always restrict our attention to initial data with compact support. This will be enough to show that the Hamiltonian is not bounded from below 
under certain conditions, although strictly speaking it is not enough to show that it is bounded from below under other conditions.

When we use the chosen form of the deSitter metric as our background metric in the Hamiltonian, we find - although the reader will have to take this on trust, since we did not give the explicit form of the Hamiltonian in an arbitrary background - that

$$
\begin{gathered}
H=\int \frac{1}{\sqrt{g}}\left(p^{a b} p_{a b}-\frac{1}{2} p p\right)+ \\
+\frac{\sqrt{g}}{4}\left(h \triangle h-h_{a b} \triangle h_{a b}-2 \partial_{b} h^{a b} \partial^{c} h_{a c}+2 \partial^{a} h \partial^{b} h_{a b}\right)+ \\
+\alpha\left(h p-2 h_{a b} p^{a b}\right)+\alpha^{2} \sqrt{g} h_{a b} h^{a b}+ \\
+\frac{m^{2}}{4} \sqrt{g}\left(h_{a b} h^{a b}-h^{2}\right)+\frac{2}{m^{2} \sqrt{g}} \partial_{b} p^{a b} \partial^{c} p_{a c} .
\end{gathered}
$$

The spatial background metric (which has been used to raise and lower indices) is flat but time dependent, so this is a time dependent Hamiltonian.

The constraints affect only the scalar modes:

$$
\begin{gathered}
\Psi_{1}=\sqrt{g}\left(\triangle h^{(0)}+\left(2 \alpha^{2}-m^{2}\right) h\right)+2 \alpha p=0 \\
\Psi_{2}=2 \triangle p^{\left(0^{\prime}\right)}+m^{2} p+2 \alpha \sqrt{g}\left(2 \triangle h^{(0)}-\triangle h\right)=0 .
\end{gathered}
$$

(Recall that $h \equiv h_{a}^{a}, h^{(0)} \equiv h_{a}^{(0)} a$ etc.) We now have to decide on a set of independent data, in terms of which the constraints are to be solved for the remaining data. It turns out that a convenient set of independent data is $h^{(0)}$ and

$$
p^{\prime} \equiv p^{\left(0^{\prime}\right)}+\alpha \sqrt{g}\left(2 h^{(0)}-h\right) .
$$

We now turn to the positivity properties of the Hamiltonian. In a flat spacetime it is not too difficult to show, using a decomposition of the fields into irreducible parts, that the Hamiltonain is positive semi-definite. In deSitter space on the other hand - as the reader may verify - an attempt to express the Hamiltonian as a function

$$
H=H\left(h^{(2)}, p^{(2)}, h^{(1)}, p^{(1)}, h^{(0)}, p^{\prime}\right)
$$

leads to a surprisingly lengthy calculation. Therefore we simplify matters further by choosing initial data such that $p^{\prime}=0$. Then one can show that

$$
H_{\mid p^{\prime}=0}=
$$




$$
\begin{gathered}
=\int \frac{1}{\sqrt{g}}\left(p_{a b}^{(2)}-\alpha \sqrt{g} h_{a b}^{(2)}\right)\left(p^{(2) a b}-\alpha \sqrt{g} h^{(2) a b}\right)+\frac{\sqrt{g}}{4} \partial_{c} h_{a b}^{(2)} \partial^{c} h^{(2) a b}+\frac{m^{2} \sqrt{g}}{4} h_{a b}^{(2)} h^{(2) a b}+ \\
+\frac{1}{\sqrt{g}}\left(p_{a b}^{(1)}-\alpha \sqrt{g} h_{a b}^{(1)}\right)\left(p^{(1) a b}-\alpha \sqrt{g} h^{(1) a b}\right)+\frac{1}{m^{2} \sqrt{g}} \partial_{c} p_{a b}^{(1)} \partial^{c} p^{(1) a b}+\frac{m^{2} \sqrt{g}}{4} h_{a b}^{(1)} h^{(1) a b}+ \\
+\sqrt{g}\left(\frac{3 m^{2}+2 \alpha^{2}}{8\left(m^{2}-2 \alpha^{2}\right)} \partial_{a} h^{(0)} \partial^{a} h^{(0)}+\frac{3 m^{2}}{8} h^{(0)} h^{(0)}\right)+ \\
+\alpha^{2} \sqrt{g}\left(\frac{1}{2}\left(h-h^{(0)}\right)^{2}+h^{(0)} h^{(0)}+\frac{2}{m^{2}} \partial_{a}\left(h-2 h^{(0)}\right) \partial^{a}\left(h-2 h^{(0)}\right)+\right. \\
\left.+4 \partial_{a} h^{(0)} \partial^{a} h^{(0)}\right) .
\end{gathered}
$$

By inspection of this expression, we see that there is a term among the scalar modes which can assume negative values if $3 m^{2}<6 \alpha^{2}=2 \lambda$, which proves that the Hamiltonian can not be positive semi-definite for such values of $\mathrm{m}^{2}$.

\section{ATTEMPTS TO DEFORM THE GAUGE ALGEBRA.}

Before we end this note, we will briefly consider whether it is possible to deform the algebra of the peculiar gauge symmetry which arises for $3 m^{2}=2 \lambda$. The question we ask is whether there exists a non-linear theory invariant under a non-abelian gauge group for which, in a linearized limit, the abelian gauge transformation (5) is recovered. This kind of question has been asked in the past concerning higher spin gauge algebras, and it is known to be very difficult to deform any abelian gauge algebra involving more than one derivative in such a manner [7]. (More recently, there has been progress in higher spin theory, but we will not go into this here. See ref. [8] and references therein.)

So we search for a non-linear transformation of the (somewhat schematically written) form

$$
\delta h_{\alpha \beta}=\nabla_{\alpha} \nabla_{\beta} \xi+g_{\alpha \beta} \xi+\left(\nabla^{2} \xi h\right)_{\alpha \beta} .
$$

Now one can write down a general Ansatz for the structure constants, and check whether it can obey the Jacobi identity [7]. Note that this kind of argument is very general; it is capable of ruling out also transformations much more general than that sketched here, such as transformations that involve several tensor fields in a non-diagonal fashion.

As long as the field $h_{\alpha \beta}$ carries no internal index, the only possible form that the commutator of two non-linear transformations can take is, to lowest order,

$$
\left[\delta_{\xi}, \delta_{\eta}\right]^{(0)}=\delta_{\eta} \nabla^{2} \xi-\xi \nabla^{2} \eta
$$

However, it is then easy to check that this commutator does not obey the Jacobi identity

$$
\left[\delta_{\omega},\left[\delta_{\xi}, \delta_{\eta}\right]\right]+\left[\delta_{\eta},\left[\delta_{\omega}, \delta_{\xi}\right]\right]+\left[\delta_{\xi},\left[\delta_{\eta}, \delta_{\omega}\right]\right]=0
$$


It follows that there does not exist any deformation of the abelian gauge algebra into a non-abelian algebra of the suggested type.

A little bit more generally, one may introduce an internal symmetry index $i$, which permits the Ansatz

$$
a_{1} f_{i j k} \nabla_{\gamma} \xi^{j} \nabla^{\gamma} \eta^{k}+a_{2} f_{i j k}\left(\square \xi^{j} \eta^{k}+\xi^{j} \square \eta^{k}\right)+a_{3} f_{i j k} \xi^{j} \eta^{k}
$$

for the zeroth order structure constants in eq. (36). Going through the Jacobi identity

again, with the $f_{i j k}$ 's obeying the Jacobi identity for an ordinatry Lie algebra, one finds that $a_{1}=a_{2}=0$, so this seems to be of no interest either.

We conclude that that it is unlikely that any deformation of the gauge algebra under study exists.

\section{References}

[1] A. Higuchi, Nucl. Phys. B282 (1987) 397.

[2] S. Deser and R.I. Nepomechie, Ann. Phys. 154 (1984) 396.

[3] C. Marzban, B.F. Whiting and H. van Dam, J. Math. Phys. 30 (1989) 1877.

[4] C. Aragone and S. Deser, Nuovo Cim 57B (1980) 33.

[5] H. van Dam and M. Veltman, Nucl. Phys. B22 (1970) 397.

D.G. Boulware and S. Deser, Phys. Rev. D6 (1972) 3368.

[6] U. Ottoson, Comm. Math. Phys. 8 (1968) 228.

A. Higuchi, J. Math. Phys.28 (1987) 1552.

[7] A.K.H. Bengtsson, Phys. Rev. D32 (1985) 2031.

F.A. Berends, G.J.H. Burgers and H. van Dam, Nucl. Phys. B260 (1985) 295.

[8] M.A. Vasiliev, Phys. Lett. B257 (1991) 111. 